\title{
A New Path Planning Strategy Based on Level Set Function for Layered Fabrication Processes
}

\author{
Xiaoying Hong \\ Chongqing University \\ Guiqian Xiao \\ Chongqing University \\ Yancheng Zhang \\ Chongqing University \\ Jie Zhou ( $\nabla$ zhoujie635635@163.com ) \\ Chongqing University
}

\section{Research Article}

Keywords: Additive manufacturing, Tool-path generation, Level set function, equidistant offset filling

Posted Date: June 18th, 2021

DOl: https://doi.org/10.21203/rs.3.rs-625865/v1

License: (9) This work is licensed under a Creative Commons Attribution 4.0 International License. Read Full License

Version of Record: A version of this preprint was published at The International Journal of Advanced Manufacturing Technology on November 3rd, 2021. See the published version at https://doi.org/10.1007/s00170-021-08239-0. 


\title{
A New Path Planning Strategy Based on Level Set Function for Layered Fabrication
} Processes

Xiaoying Hong ${ }^{1,2}$. Guiqian Xiao ${ }^{3,1}$. Yancheng Zhang ${ }^{3}$. Jie Zhou ${ }^{1}$

1 School of Materials Science and Engineering, Chongqing University, Chongqing 400044, China

2 Sichuan Vocational College of Information Technology, Guangyuan 628017, China

3 Chongqing Forgestamp Inteligent Equipment Co. ,Ltd, Chongqing 400000, China.

Corresponding author: Jie Zhou; E-mail: zhoujie635635@163.com

\begin{abstract}
:
In this paper, a new equidistant offset filling theory based on level set function and corresponding numerical solving algorithm based on dynamic finite difference method were proposed. Firstly, the closed curve is defined as the zero-value contour of level set function. Then, the level set equation, a partial differential equation, has been built to get the level set function, and a dynamic finite difference method has been proposed to solve the level set function. Secondly, three types of cross-sections which are simple polygon, multi-island polygon and multi-hole polygon were used to test the equidistant offset filling effect of the algorithm. The test results show that the proposed theory and algorithm in this paper can well deal with the equidistant filling problem of these cross-sections. In addition, any complex section can be equidistant offset filled by the algorithm proposed in this paper, and the calculation cost is significantly related to the number of offsets, but not directly related to the section complexity. Finally, the remanufacturing of a typical crankshaft hot forging die proves that the algorithm proposed in this paper can effectively complete the remanufacturing repair of this kind of forging die. The surface of the die is smooth, and there are no defects such as porosity and slag inclusion after machining. Compared with the new die, the manufacturing cost can be saved more than $50 \%$, and the efficiency of die manufacturing can be improved more than $60 \%$. This is due to the machining allowance of the die after additive manufacturing is very small, and it can be quickly repaired on site.
\end{abstract}

Keywords: Additive manufacturing; Tool-path generation; Level set function; equidistant offset filling

\section{Introduction}

Manufacturing industries often have to deal with equidistant filling of arbitrary sections, such as additive manufacturing (AM), 3D printing and $\mathrm{CNC}$ machining. Especially, additive manufacturing (AM) or 3D printing, which is based on layer-by-layer manufacturing approach 
instead of conventional material removal methods, has gained worldwide popularity over the past thirty years ${ }^{[1]}$. Recently, wire and arc additive manufacturing (WAAM) is gaining popularity in aerospace, automotive and biomedical industries because it can easily produce large components with lower cost and lesser time compared to other additive manufacture processes ${ }^{[2-4]}$.

In WAAM, the 3D CAD model is sliced into a set of $2 \mathrm{D}$ cross section which can be used to generate the welding paths by path planning algorithm. One of the core tasks in WAAM is the welding-path generation which guide the motion of the welding torch to fill the $2 \mathrm{D}$ cross section. There are many path planning strategies have been proposed for AM, such as contour ${ }^{[5-7]} \operatorname{raster}^{[8]}$, zigzag $^{[9]}$, spiral ${ }^{[10]}$, fractal space-filling curves ${ }^{[11-12]}$, and hybrid toolpath planning approaches ${ }^{\text {Error! }}$ Reference source not found.

Among these strategies, the most basic algorithms are linear fill which offset parallel to a given line, and contour offset fill which offset parallel to the boundary. The linear filling strategy guide the welding torch to move along a linear line, which means the welding torch need to be turned frequently ${ }^{[13]}$. These characteristics of linear filling led to more arc breaks, and discontinuous acceleration of the robot, especially for the 6-axis robot, which affects the forming quality and the life of the robot ${ }^{[15-17]}$. The spiral filling strategy can solve the problem faced by linear fill strategy, but is only suitable for some special models. In WAAM, the additive manufacture model is very complex and the spiral filling strategy is not universal. The strategy of fractal space-filling curves can reduce the number of arc break and reduce shrinkage during in WAAM process. However, the moving direction of the welding torch often changes, which also leads to poor stability of robot welding. Another better filling strategy is the contour offset filling strategy which has many advantages such as: less arc break, smooth and continuous path, continuous speed and acceleration, uniform filling. Therefore, this strategy is widely used in $\mathrm{CNC}$ machining, additive manufacturing (AM) and 3D printing. However, because of the complexity of different cross section shapes, contour offset filling strategy has high complexity ${ }^{[17-19]}$. For example, in the manufacturing industry, the cross-section shape may be: single island, multi-Island, convex polygon, concave polygon. The traditional contour offset filling strategy needs to realize the operations of polygon intersection, difference, union and vertex degradation. In addition, a polygon offset may result in several polygons. So, the contour offset filling strategy must deal with the problem of polygon divergence. The complexity of these strategy limits the application in WAAM. 
In order to avoid the complex polygon operation caused by using the offset algorithm to get the contour filling line directly, this paper develops a new path planning algorithm to generate the equidistant contour filling line without complex polygon operations. The proposed algorithm firstly transforms the two-dimensional contour offset problem into a three-dimensional initial value problem of partial differential equation. Secondly, the dynamic finite difference method (DFDM) is used to solve the partial different equation, and then the contour lines also called offsetting contour lines can be obtained by cutting the solution surface of the partial equation at zero level. Finally, different cross sections are used to test the proposed algorithm, and the results show that the proposed algorithm has many advantages , such as simple structure, high efficiency, no complicated polygon operation, and can directly use the existing mature finite difference method.

\section{Theoretical bases}

The mathematical theory of the novel contour offsetting method has been developed in this part. Firstly, the two-dimensional contour has been defined as the intersection of a surface and a plane. The construction and solution of surface is the key of the problem in this part, and the surface has been described as an implicit function. Secondly, the differential equation of the surface is proposed. Finally, the evolutionary criteria are proposed to realize the equidistant migration. When the surface is solved, the problem of contour offset is transformed into the problem of solving the contour line for the implicit function.

\subsection{Mathematical definition of closed curve}

It is well known that the filling problem of cross section belongs to two-dimensional problem. In two spatial dimensions, the lower-dimensional interface is a curve that separates $\mathfrak{R}^{2}$ into separate subdomains with nonzero areas. The boundary of the cross section is closed. For easily processing, the inner contour and outer contour are defined as clockwise and anticlockwise arrangement respectively. It is easy to propose that a two-dimensional contour can be defined as the intersection of a surface and a plane. The surface can be defined as $\phi(x, y, t)$ called the level set function which was first proposed by Osher and Sethian in 1988 to solve f solve the interface 
evolution problems in the fields of fluid computing, crystal growth, image processing ${ }^{[20]}$. In this paper, the level set function can be used to solve the equidistant offsetting problem. Particularly, the closed curve can be defined by $\phi(x, y, t)=0$, in which the curve is changing over time. Then, the next problem is to determine the expression or value of the level set function. It is obviously impossible to determine the level set function directly. A desirable method is to determine the differential equation of the level set function. The time differential expression of the equation $\phi(x, y, t)=0$ is as follows:

$$
\frac{\partial \emptyset}{\partial t}+\frac{\partial \emptyset}{\partial x} \frac{\mathrm{d} x}{\mathrm{~d} t}+\frac{\partial \emptyset}{\partial y} \frac{\mathrm{d} y}{\mathrm{~d} t}=0
$$

The above equation can be simplified as follows:

$$
\frac{\partial \emptyset}{\partial t}+v|\nabla \emptyset|=0
$$

where $v=\left(\frac{\partial \emptyset}{\partial x} \frac{\mathrm{d} x}{\mathrm{~d} t}+\frac{\partial \emptyset}{\partial y} \frac{\mathrm{d} y}{\mathrm{~d} t}\right) /|\nabla \emptyset|$, and $\nabla \emptyset$ is the gradient of the implicit function which called level set function. The gradient $\nabla \varnothing$ is perpendicular to the isocontours of $\varnothing$ and points in the direction of increasing $\emptyset$. In equidistant offsetting problem, the $v$ can be defined as 1 , which is standard Hamilton-Jacobi equation ${ }^{[21]}$.

\subsection{Signed distance function}

The implicit equation $\phi(x, y, t)=0$ is used to describe the closed curves in the previous section. In this section the signed distance functions, which are a subset of the implicit functions defined in the last chapter, was discussed. A signed distance function has been defined as follows:

$$
d(x, y, t)=\min \left(\sqrt{\left(x-x^{\prime}\right)^{2}+\left(y-y^{\prime}\right)^{2}}\right)
$$

where $x$ and $y$ represent the coordinates at any point. $x^{\prime}$ and $y^{\prime}$ represent the coordinates of any point on the boundary curve $(\phi(x, y, t)=0)$.

(a)

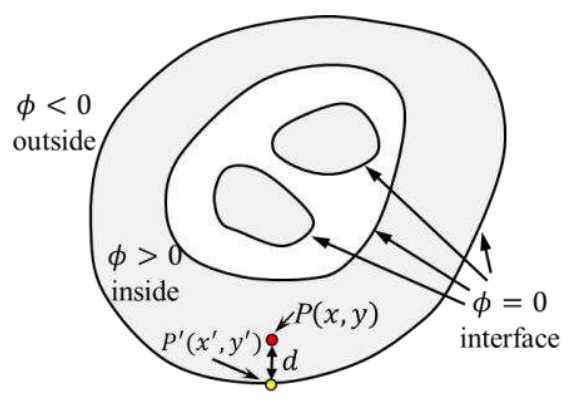

(b)

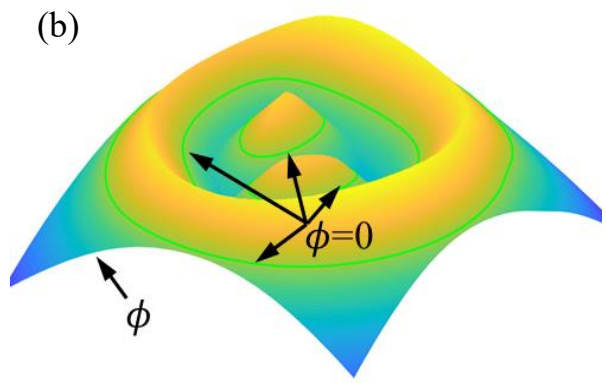

Fig.1 Implicit function $\phi$, its boundaries and distance function.

(a) Cross-section and boundary (b) Surface and boundary 
The implicit function can be defined as follows:

$$
\phi(x, y, t)= \pm d(x, y, t)
$$

As shown in Fig.1, if point $(x, y)$ is positive inside the boundary, negative outside the boundary, and zero on the boundary. If point $P^{\prime}\left(x^{\prime}, y^{\prime}\right)$ is the closest point to point $P(x, y)$, the closest point to any point on the line $P^{\prime} P$ is $P^{\prime}$. Then, the following formula can be derived:

$$
\nabla \emptyset=\left(\frac{\partial\left(\sqrt{\left(x-x^{\prime}\right)^{2}+\left(y-y^{\prime}\right)^{2}}\right)}{\partial x}, \frac{\partial\left(\sqrt{\left(x-x^{\prime}\right)^{2}+\left(y-y^{\prime}\right)^{2}}\right)}{\partial y}\right)
$$

It's easy to get $|\nabla \emptyset|=1$ from formula (5). The level set equation (2) can be simplified as follows:

$$
\frac{\partial \emptyset}{\partial t}+v=0
$$

where, $v$ is the distance that the boundary motion in unit time.

\section{Numerical algorithms}

The formulas and equations deduced in the previous part are all based on analytical theory. In practice, the section which need to be filled is very complex, so it is impossible to solve the partial differential equations by analytic method. In addition, due to the complexity of some cross-sections, the boundaries of these cross-section may not be described analytically, and these boundaries can only be represented by a set of points arranged clockwise or counterclockwise. Therefore, the following sections discuss the numerical algorithm required to solve the equidistant offset filling according to Eq. (6) in practical engineering.

\subsection{Auxiliary algorithms}

\subsubsection{Judgment algorithm of point in polygon}

It is necessary to identify whether a point is within the polygon because of the introduction of the signed distance function. There are many algorithms to identify whether a point is within the polygon, such as area summation algorithm, angle summation algorithm and ray algorithm. In area summation algorithm, the target point is inside the polygon if the sum of the triangle area composed of the target point and each edge of the polygon is equal to area of the polygon. In angle summation algorithm, the target point is inside the polygon if the sum of angle between the target point and all 
the edges is equal to 360 degrees. In ray algorithm, a ray is emitted from the target point. If the number of the points which intersect the edges of the polygon is odd, the target point is inside the polygon.

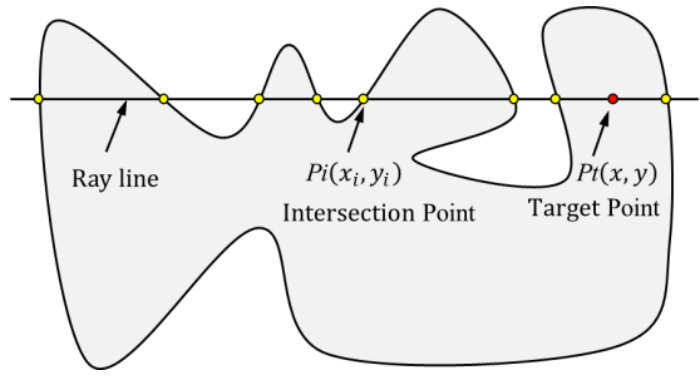

Fig.2 Schematic diagram of ray algorithm

In this paper, the last algorithm has been used. The detailed steps are as follows: compare the $y$ coordinate of the target point with each point of the polygon to get a list of intersection points between the horizontal line where the target point is and the polygon edge. If the number of the intersection points on both sides of the target point is odd, the target point is inside the polygon, otherwise it is outside the polygon. In the example as shown in Fig.2, there are seven intersections on the left and one intersection on the right of the target point. They are all odd numbers, so the point is in the polygon.

\subsubsection{Judgment algorithm of polygon direction}

It is necessary to judge whether the polygon is clockwise or counterclockwise when the zerosolution contour line of the implicit function is obtained, and the inner and outer contour judgment algorithm is needed at this time. A general method to judge the direction of a complex polygon is curve integral, according to Green's formula:

$$
\iint_{D}\left(\frac{\partial Q}{\partial x}-\frac{\partial P}{\partial y}\right) d x d y=\oint_{\partial D^{+}}(P d x+Q d y)
$$

where $\partial D^{+}$is the counterclockwise boundary of region $D$. Green's formula reveals the relationship between double integrals in a plane region and plane curve integrals. If we take the integrand $Q=x$ and $P=0$, then the following equation is derived according to Green's formula:

$$
s=\iint_{D} \mathrm{~d} x d y=\oint_{\partial D^{+}} x \mathrm{~d} y
$$

where $S$ represents the area of region D. If the integral path is counterclockwise, the integral is positive. Otherwise, the integral is negative. Therefore, by calculating the positive and negative 
values of the curve integral, we can determine whether the polygon boundary is clockwise or counterclockwise. For the discrete polygon boundary, the discrete formula can be used to calculate the curve integral:

$$
s=\oint_{\partial D^{+}} x d y \approx \frac{1}{2} \sum_{i=1}^{n}\left(x_{i+1}+x_{i}\right)\left(y_{i+1}-y_{i}\right)
$$

where $n$ represents the number of discrete points of the polygon, $x_{i}$ and $y_{i}$ are coordinates of the $i-t h$ point on the boundary of the polygon. The advantage is that no matter how complex the polygon is, it can judge the sequence and inverse relationship of the polygon under the linear complexity, and the programming is relatively simple by using Green's formula.

\subsection{3 judgment algorithm of Inner or outer boundary}

A cross-section may be composed of several groups of polygons, so how to automatically judge the inside and outside of a polygon is very important. A simple method is to calculate the number of times a contour is included. If a contour is included even times, it is an outer contour, otherwise it is an inner contour.

(a)

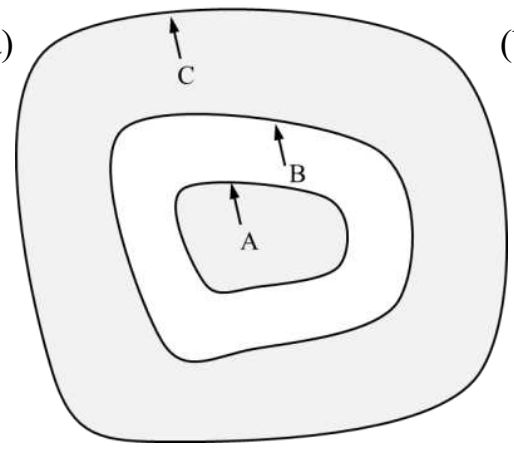

(b)

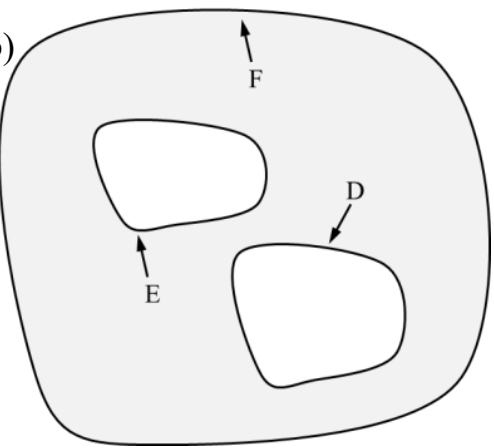

Fig.3 Schematic diagram of the relationship between the inner and outer contours being included. (a) single island cross-section (b) two holes cross-section.

As shown in Fig.3, A, C and F are outer contours, which has been included even number of times. B, E and D are inner contours, which has been included old number of times. Whether a target polygon is included in another polygon can be done as follows: traverse all vertices of the target polygon to determine whether all vertices are included in another polygon. If all vertices are included in another polygon, then the polygon is included. 


\subsection{Dynamic finite difference method}

Partial differential equation (6) is the core of equidistant offset filling. It is well known that the numerical solution of Partial Differential Equation (PDE) can be solved by the Dynamic Finite Difference Method (DFDM). The level set function $\phi(x, y, t)$ is discretized into a sequence in the time dimension and into a two-dimensional grid in the space dimension. Then, $\phi(x, y, t)$ is defined at every grid point on Cartesian grid. Updating $\phi$ in time consists of finding new values of $\phi$ at every grid point after some time increment $\Delta t$. A simple first-order accurate method for the time discretization of equation (6) is the forward Euler method given by

$$
\frac{\phi\left(x_{i}, y_{j}, t_{k+1}\right)-\phi\left(x_{i}, y_{j}, t_{k}\right)}{\Delta t}+v\left(x_{i}, y_{j}, t_{k}\right)=0
$$

where $t_{k+1}=t_{k}+\Delta t, x_{i+1}=x_{i}+\Delta x$ and $y_{i+1}=y_{i}+\Delta y . \Delta t, \Delta x$ and $\Delta y$ are increments in time, $x$ and $y$ respectively, the smaller these values are, the higher the solution precision is. In addition, $i=1,2, \ldots ., M . j=1,2, \ldots, N . \quad k=1,2, \ldots, W$. The initial value of the level function $\phi$ can be calculated by signed distance function. In particular, considering the equidistant offset, the points on the contour are offset at the same velocity, so $v$ is a constant independent of time and position. Then, the solution algorithm can be simply described as follows:

$$
\left\{\begin{array}{l}
\phi\left(x_{i}, y_{j}, t_{k+1}\right)=v \Delta t+\phi\left(x_{i}, y_{j}, t_{k}\right) \\
\phi\left(x_{i}, y_{j}, t_{1}\right)=f\left(x_{i}, y_{j}\right)
\end{array}\right.
$$

where, $f\left(x_{i}, y_{j}\right)$ is the discrete signed distance function in which the auxiliary algorithms should be used to calculated the signed minimum distance. The judgment algorithm of Inner or outer boundary and the judgment algorithm of polygon direction are used together to calculate the inside and outside of the boundary, and the judgment algorithm of point in polygon is used to determine the positive and negative. In general, the detailed algorithm flow for solving Eq. (6) or Eq. (11) is as follows: 


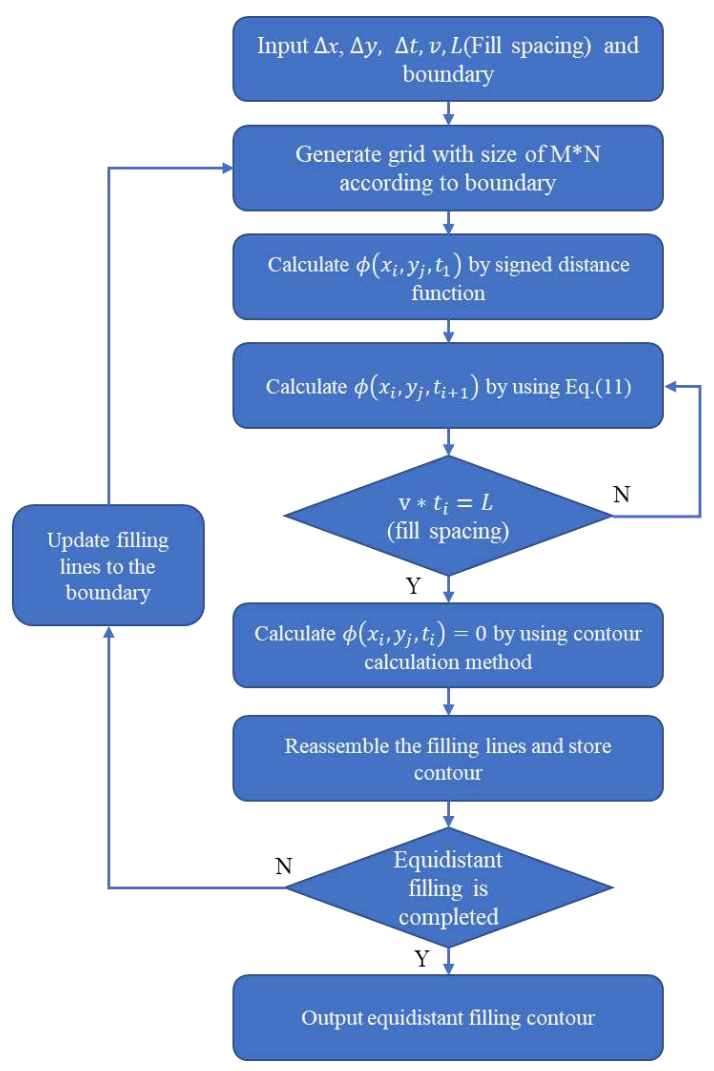

Fig.4 Flow chart of equidistant offset filling algorithm.

As shown in Fig.4, the algorithm is a dynamic FDM algorithm, which updates the boundary and initializes the level set function every time the filling line with specified spacing is obtained. This is due to the discretization of the boundary, spatial dimension and time dimension, the $|\nabla \emptyset|$ is not exactly equal to one. If all the filling lines are calculated statically, only once solving the partial differential equation, the filling lines may be distorted.

\subsection{Numerical tests}

The traditional offsetting algorithm based on geometric processing is easy to get a set of equidistant filling lines for simple convex polygon. However, it is very difficult to obtain equidistant offset filling lines for complex cross-sections, such as multi-islands, multi-holes and concave polygon. This section discusses the equidistant offset filling algorithm for any complex crosssection by using the algorithm described in this article.

\subsubsection{Simple polygon}

In order to test the effectiveness of the algorithm, two simple polygons are selected for the 
offset fill test. The offset fill effect of square, circle is shown in the figure 5. Two-dimensional space is discretized into $400 * 400$ grid in these cases, and the offset distances of the three polygons in Fig.5 are $1 \mathrm{~mm}$ and $1 \mathrm{~mm}$ respectively.
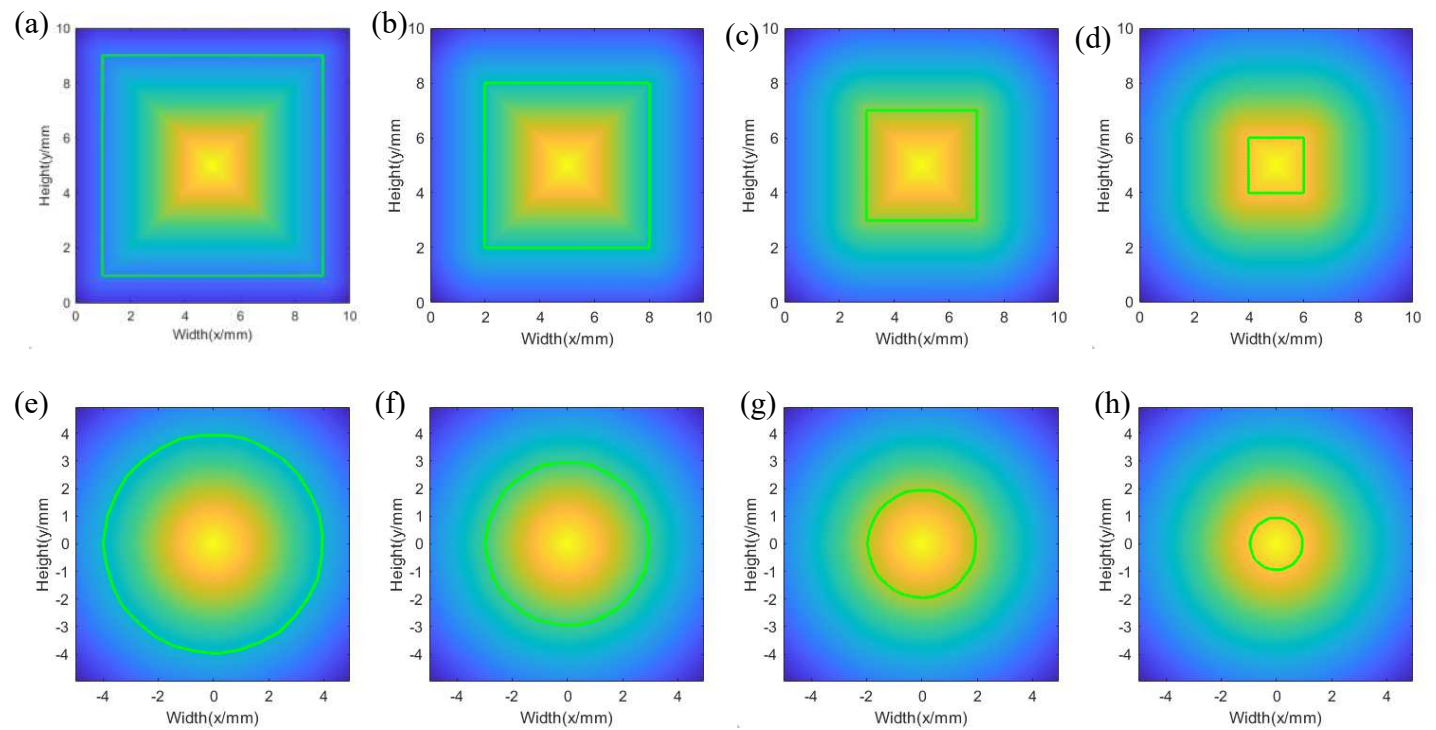

Fig.5 Offset lines and $\phi$ function values with different offset distances for two different polygons

(a) Square offset by $1 \mathrm{~mm}$ (b) Square offset by $2 \mathrm{~mm}$ (c) Square offset by $3 \mathrm{~mm}$ (d) Square offset by $4 \mathrm{~mm}$ (e)

Circle offset by $1 \mathrm{~mm}$ (f) Circle offset by $2 \mathrm{~mm}$ (g) Circle offset by $3 \mathrm{~mm}$ (h) Circle offset by $4 \mathrm{~mm}$

When the iteration satisfies $v * t_{i}=L$, the $\phi$ function are re-initialized and the iteration starts again until the polygon is completely filled. The value of level set function $\phi$ has only one extreme value and is different after each initialization. This is because for a convex polygon section with only one boundary, the polygon does not split during the offset process, that is, multiple polygons will not be generated by one polygon offset.
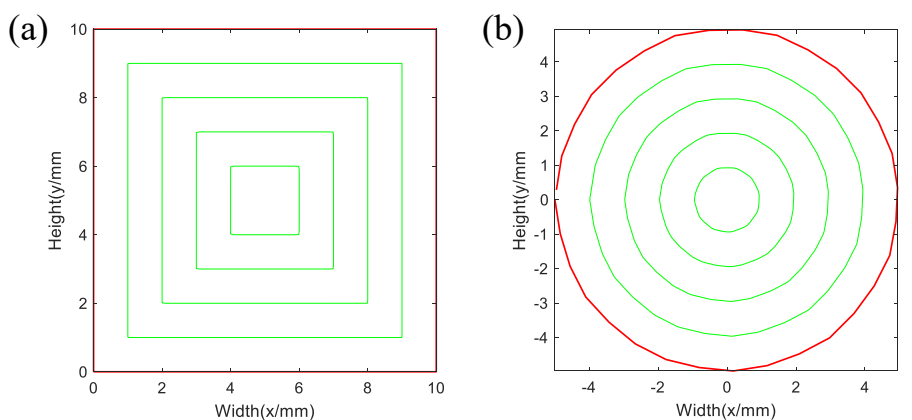

Fig.6 Equidistant offset filling results of three simple polygons (a) Square (b) Circle

The filling effect of three simple polygons is shown in Fig.5. The algorithm introduced in this paper can quickly deal with the offset filling of simple polygon. When the level set function $\phi$ does not intersect with the plane described by $\mathrm{z}=0$, the filling ends. 


\subsubsection{Multi-island polygon}

As shown in Fig. 7, the algorithm designed in this paper can also be applied to the equidistant offset filling calculation for multi-island cross-section with three outer boundary and one inner boundary. In the case of multi-island cross-section, the level set function has multiple extreme values, and the level set function varies greatly. So, the dynamic finite difference method is very necessary to solve the level set equation.
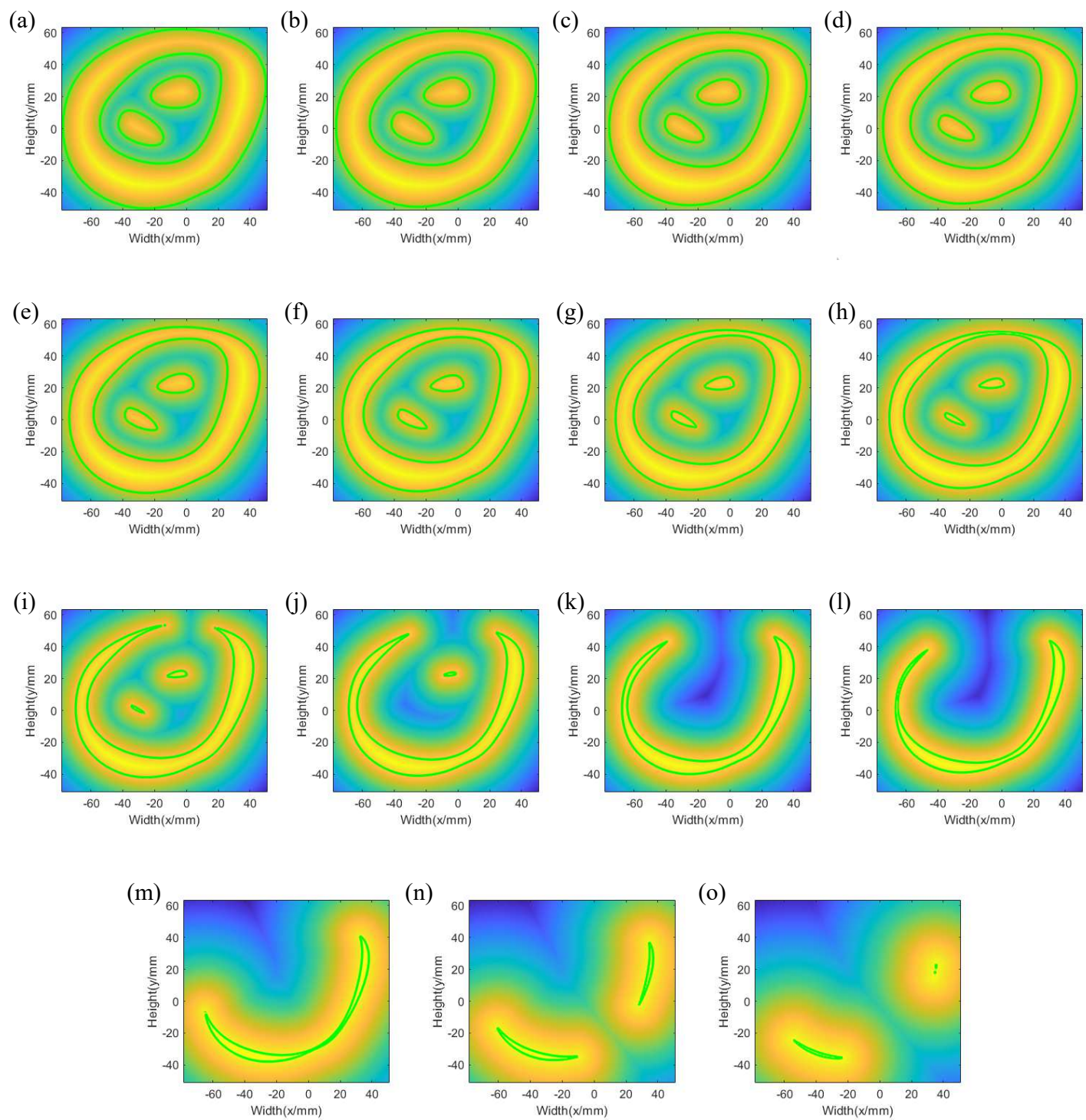

Fig.7 The multi-island cross-section offset curves and level set functions at different offset distances

(a) Offsetting distance $1 \mathrm{~mm}$ (b) Offsetting distance $2 \mathrm{~mm}$ (c) Offsetting distance $3 \mathrm{~mm}$ (d) Offsetting distance $4 \mathrm{~mm}$ (e) Offsetting distance $5 \mathrm{~mm}$ (f) Offsetting distance $6 \mathrm{~mm}$ (g) Offsetting distance $7 \mathrm{~mm}$ (h) Offsetting distance

$8 \mathrm{~mm}$ (i) Offsetting distance 9mm (j) Offsetting distance 10mm (k) Offsetting distance $11 \mathrm{~mm}$ (l) Offsetting distance $12 \mathrm{~mm}(\mathrm{~m})$ Offsetting distance $13 \mathrm{~mm}$ (m) Offsetting distance $14 \mathrm{~mm}$ (n) Offsetting distance 15mm (o)

Offsetting distance $16 \mathrm{~mm}$ 
In this cases, two-dimensional space is discretized into $1000 * 1000$ grid, and the offset distance is defined as $2 \mathrm{~mm}$. As shown in Fig.8, the number of polygons obtained by the offsetting is changed in the process of equidistant offsetting. Fortunately, in this algorithm, no special operation is required whether polygons are added, subtracted or disappeared, and the algorithm can automatically evolve the polygon by taking the contour of $\phi=0$.

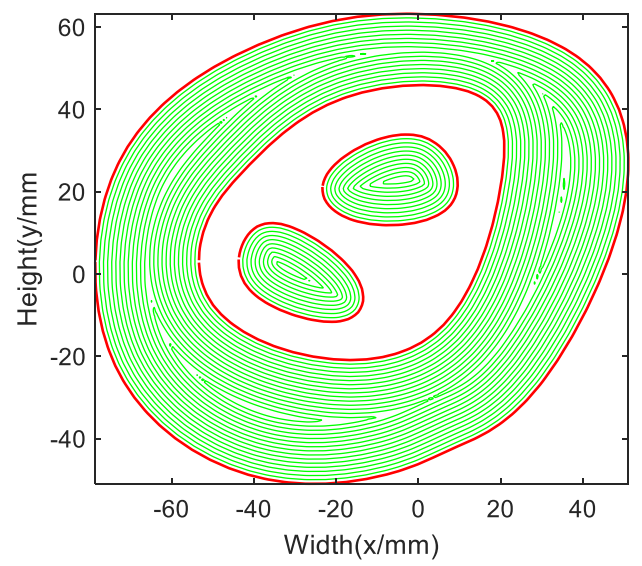

Fig.8 The equidistant offset filling contours for multi-island cross-section

\subsubsection{Multi-hole polygon}

Another complex situation is the multi-hole cross-section as shown in Fig.9 and Fig.10. There are five inner boundary and one outer boundary, which results in the level set function having multiple extreme values. In this cases, two-dimensional space is discretized into $1000 * 1000$ grid, and the offset distance is defined as $1 \mathrm{~mm}$.

(a)

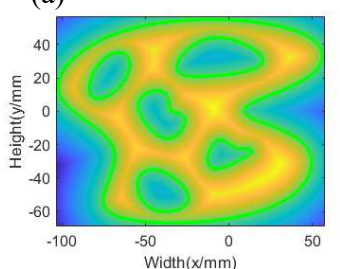

(e)

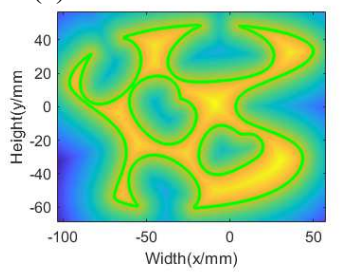

(i) (b)

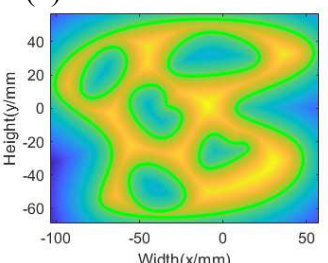

(f)

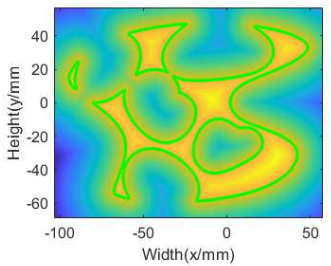

(j) (c)

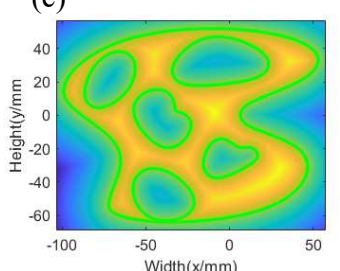

(g)

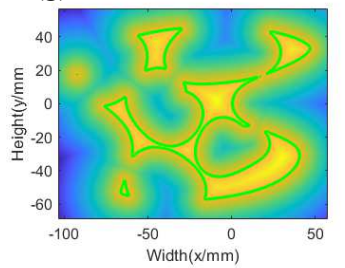

(k) (d)

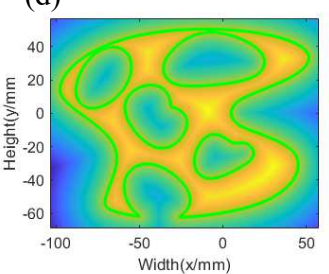

(h)

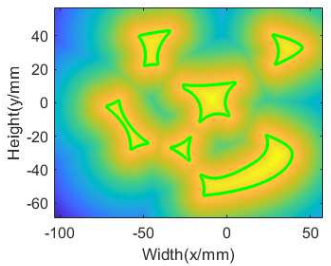

(1) 

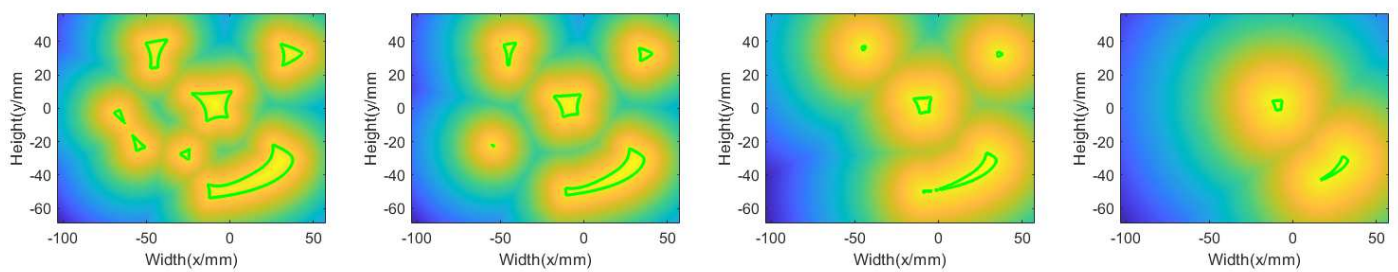

Fig.9 The multi-hole cross-section offset curves and level set functions at different offset distances

(a) Offsetting distance $1.5 \mathrm{~mm}$ (b) Offsetting distance $3 \mathrm{~mm}$ (c) Offsetting distance $4.5 \mathrm{~mm}$ (d) Offsetting distance $6 \mathrm{~mm}$ (e) Offsetting distance $7.5 \mathrm{~mm}$ (f) Offsetting distance $9 \mathrm{~mm}$ (g) Offsetting distance $10.5 \mathrm{~mm}$ (h) Offsetting distance $12 \mathrm{~mm}$ (i) Offsetting distance $12.5 \mathrm{~mm}$

The level set function varies with the boundary, and the polygon boundary changes are much more complex than in the previous multi-island case. The algorithm presented in this paper can easily handle the filling problem of complex cross-section, no matter how complex the cross sections are. In addition, the complexity of the algorithm is related to the number of offset and independent of the shape of the cross-section.

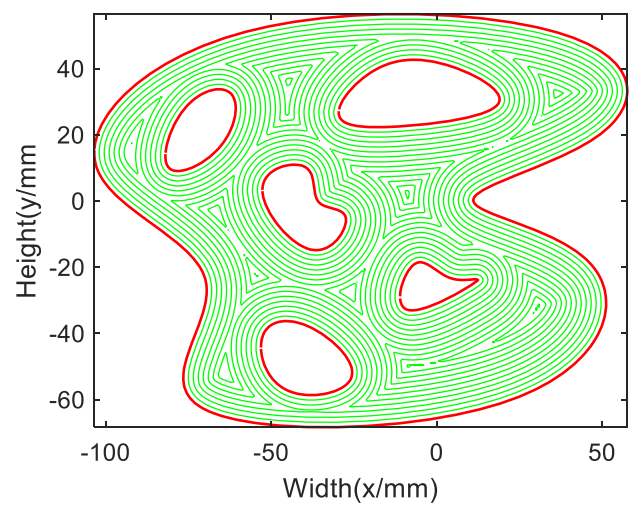

Fig.10 The equidistant offset filling contours for multi-hole cross-section

As is shown in Fig.10, the equidistant filling line shrinks from the outside to the inside, which is important for arc fuse additive remanufacturing processes because the welding torch can only be deflected toward the center or it may hit the base material.

\section{Additive Manufacture Experiment}

In this section, a typical hot forging die is used for additive remanufacturing to analysis the effectiveness of the algorithm described above. The welding process parameters are as follows: 
voltage is $24.5 \mathrm{~V}$, current is $200 \mathrm{~A}$, wire feeding speed is $600 \mathrm{~mm} / \mathrm{min}$ and welding speed is $450 \mathrm{~mm} / \mathrm{min}$. Under these parameters, the width and height of the weld are $9 \mathrm{~mm}$ and $3 \mathrm{~mm}$ respectively. Therefore, the slicing height is $3 \mathrm{~mm}$ and the offsetting distance is $4.5 \mathrm{~mm}$. A typical cross-section of crankshaft die is shown in Fig.11.

(a)

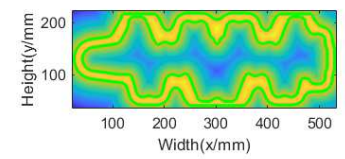

(b)

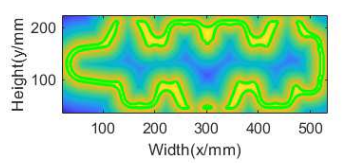

(c)

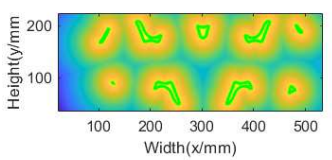

(d)

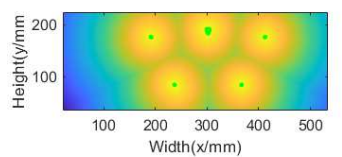

Fig.11 Level set function and equidistant offset boundary of a typical cross-section

(a) Offsetting distance $4.5 \mathrm{~mm}$ (b) Offsetting distance $9 \mathrm{~mm}$ (c) Offsetting distance $13.5 \mathrm{~mm}$ (d) Offsetting distance $18 \mathrm{~mm}$

The section is thin, so it is difficult to fill it evenly by traditional filling algorithm. This section has only one outer boundary and one inner boundary. When the offsetting distance is $9 \mathrm{~mm}$, the boundary will split into five outer boundaries. When offsetting distance is $13.5 \mathrm{~mm}$, the boundary continues to divide into nine outer boundaries. Finally, when the filling is completed, some boundaries disappear, leaving only five small outer boundaries.

(a)

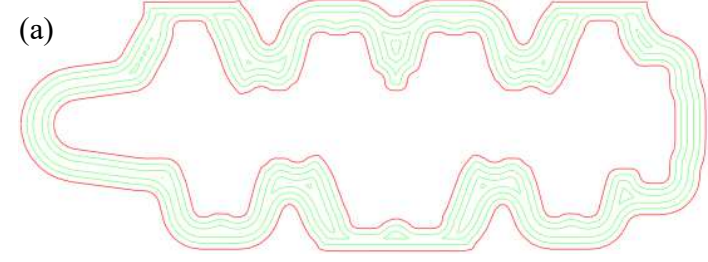

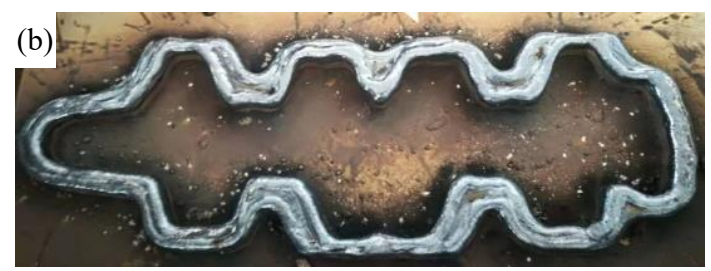

Fig.12 Equidistant offset fill contours and welding test.

(a) Equidistant offset fill contours (b) Welding sample

The offsetting filling and welding filling effects of this section are shown in Fig.12. The section is relatively smooth after welding. Although there are small pits in local areas, these small pits are filled out as the current layer is covered by the next layer throughout the additive manufacturing process. Because the size of the section cannot be divided by the width of the weld, no matter what algorithm is used, there will be overfilling or underfilling in local areas. Fortunately, these areas are very small and different layer positions are different, so they can be filled automatically in the additive manufacturing process. 

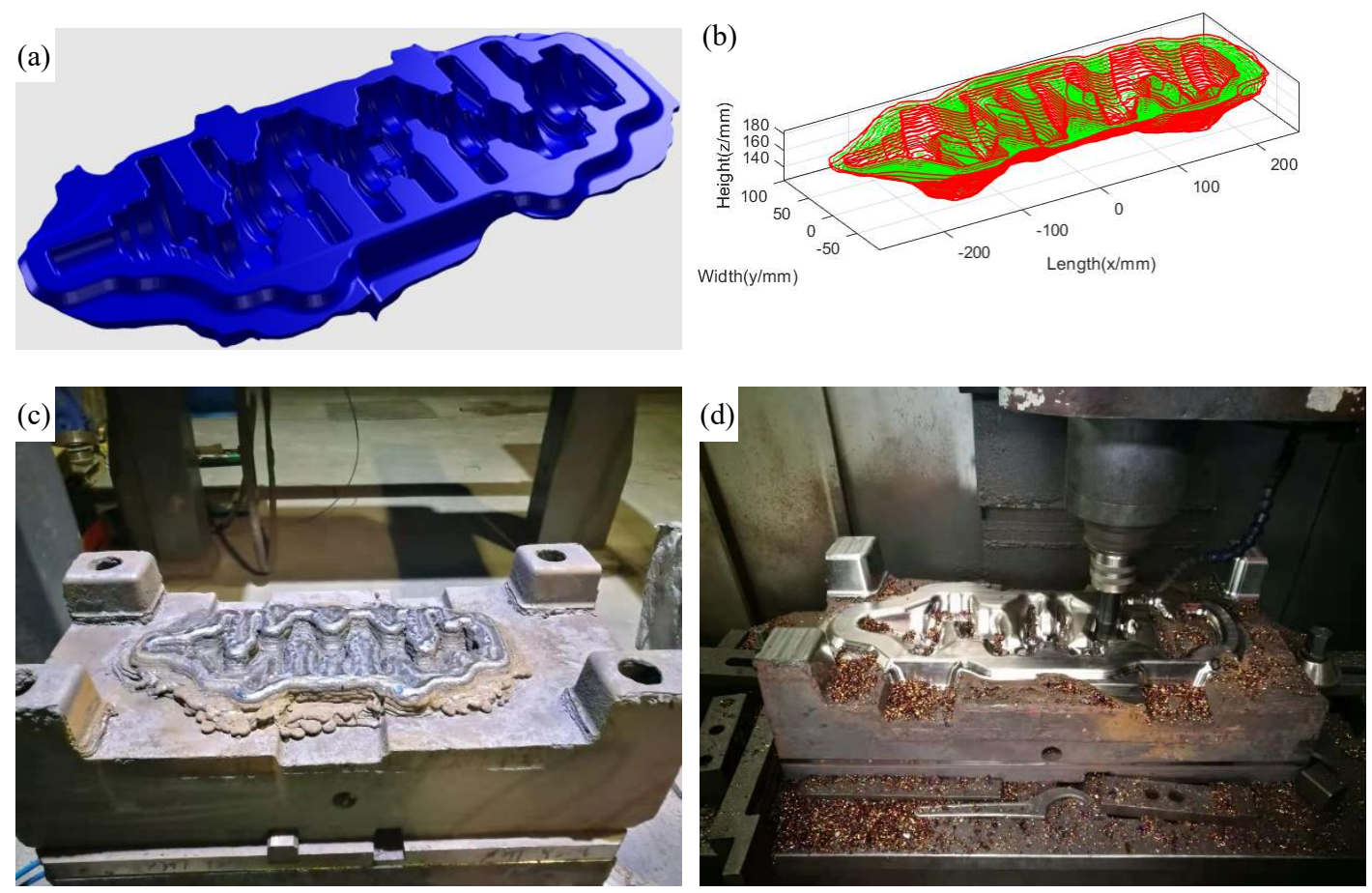

Fig.13 Wire arc additive remanufacture process of crankshaft hot forging die

(a) Target model (b) Slicing and equidistant offset filling (c) Additive welding (d) Machining

As shown in Fig.13, the process of wire arc additive manufacturing includes: extracting target model, slicing, equidistant offset filling, welding and machining. The target model is a 3D model obtained by scanning or modeling, which needs additive manufacturing. In this paper, a target model of crankshaft hot forging die has been shown in Fig.13a. This target model is very complex, which has many complex surfaces and curves. The equidistant offset filling which is the key task of this process, which directly affects the welding accuracy and quality. As shown in Fig.13bc, the welding surface is smooth and the shape accuracy is high. At the edge of the die base, there is a welding tumor. This is due to excessive welding heat input and outflow of weld metal, but does not affect the die quality. As shown in Fig.13d, the surface of the die is smooth, and there are no defects such as porosity and slag inclusion after machining. Compared with the new die, the cost can be saved more than $50 \%$, and the efficiency of die manufacturing can be improved more than $60 \%$. This is due to the machining allowance of the die after additive manufacturing is very small, and it can be quickly repaired on site. 


\section{5 conclusions}

(1) In this paper, a new equidistant offset filling theory based on level set function has been proposed, and the corresponding numerical solving algorithm based on dynamic finite difference method has been developed. Firstly, the plane closed curve is defined as the zero-value contour of implicit function. Then, the level set equation, a partial differential equation, has been developed to get this implicit function. Finally, a dynamic finite difference method has been proposed to solve the level set function.

(2) Three types of cross-sections which are simple polygon, multi-island polygon and multihole polygon are used to test the equidistant offset filling effect of the algorithm. The test results show that the algorithm in this paper can well deal with the equidistant filling problem of these sections. In addition, any complex section can be equidistant offset filled by the algorithm proposed in this paper, and the calculation cost is significantly related to the number of offsets, but not directly related to the section complexity.

(3) The remanufacturing of a typical crankshaft hot forging die proves that the algorithm proposed in this paper can effectively complete the remanufacturing repair of this kind of forging die. The surface of the die is smooth, and there are no defects such as porosity and slag inclusion after machining. Compared with the new die, the manufacturing cost can be saved more than $50 \%$, and the efficiency of die manufacturing can be improved more than $60 \%$. This is due to the machining allowance of the die after additive manufacturing is very small, and it can be quickly repaired on site. 


\section{Declarations}

Ethical approval: Not applicable

Consent to Participate: Not applicable

Consent to Publish: All the authors gave their consent for the manuscript to be published.

Authors Contributions: Xiaoying Hong put forward the relevant algorithm and ideas, and wrote the article. Guiqian Xiao wrote the software and developed the hardware and carried out experiments. Yancheng Zhang did the welding process parameter experiment. Jie Zhou guided and reviewed the whole process.

Funding: This research is supported by National Natural Science Foundation of China (No.52075058) and by Postdoctoral Science Foundation of Chongqing Natural Science Foundation (No. cstc2020jcyj-bshX0006).

Competing Interests: No conflict of interest exists in the submission of this manuscript, and manuscript is approved by all authors for publication. I would like to declare on behalf of my coauthors that the work was original research that has not been published previously.

Availability of data and materials: All data generated or analyzed during this study are included in this published article.

\section{Reference}

[1] Ding D , Pan Z, Cuiuri D, Li H (2015) A practical path planning methodology for wire and arc additive manufacturing of thin-walled structures. Robotics \& Computer Integrated Manufacturing, 34(aug.): 8-19.

[2] Singh S , Sharma S K , Rathod D W (2021) A review on process planning strategies and challenges of WAAM. Materials Today: Proceedings, 2021(4).

[3] Yaseer A, Chen H (2021) A Review of Path Planning for Wire Arc Additive Manufacturing (WAAM). Journal of Advanced Manufacturing Systems.

[4] Karunakaran K P , Suryakumar S , Pushpa V , Akula S (2010) Low cost integration of additive and subtractive processes for hybrid layered manufacturing. Robotics \& Computer Integrated Manufacturing , 26(5): 490-499.

[5] Farouki R T, Koenig T, Tarabanis K A, Korein JU , Batchelader JS (1995) Path planning with offset curves for layered fabrication processes. Journal of Manufacturing Systems, 14(5): 355-368.

[6] Yang Y , Loh H T , Fuh J , Wang Y (2002) Equidistant path generation for improving scanning efficiency in layered manufacturing. Rapid Prototyping Journal, 8(1): 30-37.

[7] Li H , Dong Z, Vickers GW (1994) Optimal toolpath pattern identification for single island, sculptured part rough machining using fuzzy pattern analysis. Computer-Aided Design, 26(11): 787-795.

[8] Dunlavey, Michael R (1983) Efficient polygon-filling algorithms for raster displays. ACM Transactions on Graphics, 2(4):264-273.

[9] S. C , Park, B. K. , Choi (2000) Tool-path planning for direction-parallel area milling. 
Computer-Aided Design, 32(1): 17-25.

[10] Wang H , Jang P , Stori J A (2001) A Metric-Based Approach to Two-Dimensional (2D) ToolPath Optimization for High-Speed Machining. American Society of Mechanical Engineers.

[11] Kulkarni P , Marsan A , Dutta D (2000) A review of process planning techniques in layered manufacturing. Rapid Prototyping Journal, 6(1): 18-35.

[12] Konrad, C (2011) Two-constraint domain decomposition with space filling curves. Parallel Computing, 37(4-5), 203-216.

[13] Dwivedi R , Kovacevic R (2004) Automated torch path planning using polygon subdivision for solid freeform fabrication based on welding. Journal of Manufacturing Systems, 23(4):278291.

[14] Jin G Q , Li W D , Gao L (2013) An adaptive process planning approach of rapid prototyping and manufacturing. Robotics \& Computer Integrated Manufacturing, 29(1): 23-38.

[15] Dunlavey, Michael R (1983) Efficient polygon-filling algorithms for raster displays. ACM Transactions on Graphics, 2(4): 264-273.

[16] Rajan V T, Srinivasan V , Tarabanis K A (2001) The optimal zig-zag direction for filling a two dimensional region. Rapid Prototyping Journal, 7(5):231-241.

[17] S. C. , Park, B. K. , Choi. (2000). Tool-path planning for direction-parallel area milling. Computer-Aided Design, 32(1), 17-25.

[18] Sang C P , Yun C C , Choi B K (2003) Contour-parallel offset machining without toolretractions. Computer-Aided Design, 35(9): 841-849.

[19] Mao L , Gao F , Yuan Z , Chen X , Benke WU (2017) Filling algorithm of two-dimensional contour in selective laser melting molding. Journal of Hefei University of Technology.

[20] Osher S , Sethian J A (1988) Fronts propagating with curvature-dependent speed: Algorithms based on Hamilton-Jacobi formulations. Journal of Computational Physics, 79( 1):12-49.

[21] Crandall M , Lions, Pierre L (1983). Viscosity solutions of hamilton-jacobi equations. Transactions of the American Mathematical Society, 277(1), 1-42. 